\title{
Glucose regulates protein interactions within the yeast SNF1 protein kinase complex
}

\author{
Rong Jiang' and Marian Carlson ${ }^{1,2,3}$ \\ Departments of ${ }^{1}$ Genetics and Development and ${ }^{2}$ Microbiology, Columbia University, New York, New York 10032 USA
}

\begin{abstract}
The SNF1 protein kinase is broadly conserved in eukaryotes and has been implicated in responses to environmental and nutritional stress. In yeast, the SNF1 kinase has a central role in the response to glucose starvation. SNF1 is associated with its activating subunit, SNF4, and other proteins in complexes. Using the two-hybrid system, we show that interaction between SNF1 and SNF4 is strongly regulated by the glucose signal. Moreover, this interaction is appropriately affected by mutations in regulators, including protein phosphatase 1. We show that SNF4 binds to the SNF1 regulatory domain in low glucose, whereas in high glucose the regulatory domain binds to the kinase domain of SNF1 itself. Genetic analysis further suggests that the SNF1 regulatory domain autoinhibits the kinase activity and that in low glucose SNF4 antagonizes this inhibition. Finally, these interactions have been conserved from yeast to plants, indicating that homologs of the SNF1 kinase complex respond to regulatory signals by analogous mechanisms.
\end{abstract}

[Key Words: SNF1 protein kinase; autoinhibition; glucose signal; S. cerevisiae]

Received October 11, 1996; revised version accepted November 6, 1996.

The SNF1 (CAT1, CCR1) protein kinase was first identified genetically in the yeast Saccharomyces cerevisiae and is now known to be conserved in eukaryotes ranging from plants to mammals. SNF1 and its homologs have been implicated in cellular responses to a variety of nutritional and environmental stresses. In S. cerevisiae, the SNFl serine/threonine kinase is essential for the response to glucose starvation and is required for transcription of glucose-repressed genes, including genes involved in alternate carbon source utilization, respiration, and gluconeogenesis (Celenza and Carlson 1986; Schuller and Entian 1987). SNF1 is also necessary for sporulation, glycogen storage, thermotolerance, and peroxisome biogenesis (Thompson-Jaeger et al. 1991; Simon et al. 1992).

In mammals, AMP-activated protein kinase (AMPK) is a sequence homolog of SNF1 (Aguan et al. 1994; Carling et al. 1994; Mitchelhill et al. 1994; Gao et al. 1995) and has a similar substrate recognition motif (Dale et al. 1995). AMPK is involved in cellular stress responses (Corton et al. 1994; Hardie 1994) and regulates key enzymes in cholesterol and fatty acid biosynthesis, HMGCoA reductase and acetyl-CoA carboxylase (Hardie 1992). It has been proposed that AMPK protects cells against environmental stresses that deplete ATP, such as heat shock, hypoxia, and oxidative stress; AMPK is activated by the elevated AMP to ATP ratio and inhibits biosynthetic pathways, thereby preserving ATP for es-

\footnotetext{
${ }^{3}$ Corresponding author.
}

sential cellular processes. Interestingly, SNF1 phosphorylates and inactivates acetyl-CoA carboxylase, suggesting that this regulatory role is conserved in yeast (Mitchelhill et al. 1994; Woods et al. 1994).

SNF1 homologs have also been cloned from a variety of plants, including rye, tobacco, Arabidopsis, and barley (Alderson et al. 1991; Halford et al. 1992; Le Guen et al. 1992; Muranaka et al. 1994). These plant kinases appear to be functional homologs of SNF1, as plant genes complement the snf1 mutation in yeast (Alderson et al. 1991; Muranaka et al. 1994). Other protein kinases related to SNF1 include PAR-1 of Caenorhabditis elegans (Guo and Kemphues 1995), mouse Msk (Ruiz et al. 1994), and human p78, a protein lost in pancreatic carcinoma cells (Hanks and Hunter 1995).

In yeast cells, the SNF1 kinase is complexed with other proteins, including the activating subunit SNF4 (CAT3) and the SIP1, SIP2, and GAL83 proteins (Celenza et al. 1989; Yang et al. 1992, 1994). Genetic and biochemical evidence indicates that SNF4 activates the SNF1 kinase: snf4 mutants have phenotypes similar to snf1 mutants (Schuller and Entian 1988; Celenza et al. 1989) and lack SNF1 catalytic activity in vitro (Celenza and Carlson 1989; Woods et al. 1994). SIP1, SIP2, and GAL83 constitute a family of related proteins, and genetic evidence suggests that these proteins are alternate members of the kinase complex, possibly serving as adaptors that mediate interaction of SNF1 with specific targets (Yang et al. 1992, 1994; Erickson and Johnston 1993). Mammalian homologs of SNF4 and SIP2 are associated with AMPK (Stapleton et al. 1994; Woods et al. 
1996), indicating that these components of the SNF1 kinase complex have counterparts in other eukaryotes.

Here we address a key question: How is the SNFl kinase activity regulated? The central function of SNF1 in the response to glucose starvation suggests that its kinase activity should be controlled by the glucose signal. SNF1 is required to derepress expression of many genes in glucose-starved cells, and the increase in expression is in many cases dramatic (>100-fold) (Johnston and Carlson 1992). However, only a two- to threefold increase in SNFl kinase activity during glucose starvation was detected by assaying phosphorylation of a synthetic peptide substrate (Woods et al. 1994), and immune complex kinase assays revealed only minor changes in SNF1 activity (Estruch et al. 1992). Moreover, the known components of the kinase complex are associated with SNF1 in both high and low glucose, as judged by coimmunoprecipitation and copurification (Celenza et al. 1989; Estruch et al. 1992; Mitchelhill et al. 1994; Stapleton et al. 1994; Yang et al. 1994). Immunofluorescence studies showed no change in subcellular localization of SNF1 and SNF4; both proteins are distributed throughout the cell, although SNF4 is partially localized to the nucleus (Celenza and Carlson 1986; Celenza et al. 1989).

In this work, we have used the two-hybrid system (Fields and Song 1989) to monitor the interaction between SNF1 and SNF4 in vivo. Using DNA-binding and activation domain fusions to SNF1 and SNF4, we assayed activation of a target promoter. We report that the SNF1-SNF4 interaction is strongly regulated by the glucose signal. Analysis of the effects of regulatory mutations confirmed the functional relevance of this assay and revealed a role for protein phosphatase 1 (PP1) in modulating this interaction. To understand the two-hybrid results, we mapped sites of protein interaction. We show that SNF4 and the SNF1 kinase domain interact with overlapping sites in the SNFl regulatory domain, with opposite regulation by the glucose signal. These findings and genetic studies suggest that SNF4 antagonizes autoinhibition of the kinase activity by the regulatory domain. We propose that the two-hybrid interactions reflect regulated interactions of the native proteins within the kinase complex. Finally, we present evidence that these interactions have been conserved in evolution.

\section{Results}

Interaction of SNF1 and SNF4 in the two-hybrid system is regulated by glucose

We used the two-hybrid system (Fields and Song 1989) to monitor interaction between SNF1 and SNF4 proteins fused to different DNA-binding and activation domains and expressed from the $A D H 1$ promoter (Fig. 1A). In all cases, SNF1 and SNF4 interacted strongly only in glucose-deprived cells, yielding roughly 100-fold greater $\beta$-galactosidase activity than in high glucose. The same pattern of glucose-regulated interaction was observed using fusions to GAL4 (GBD) or LexA DNA-binding do-
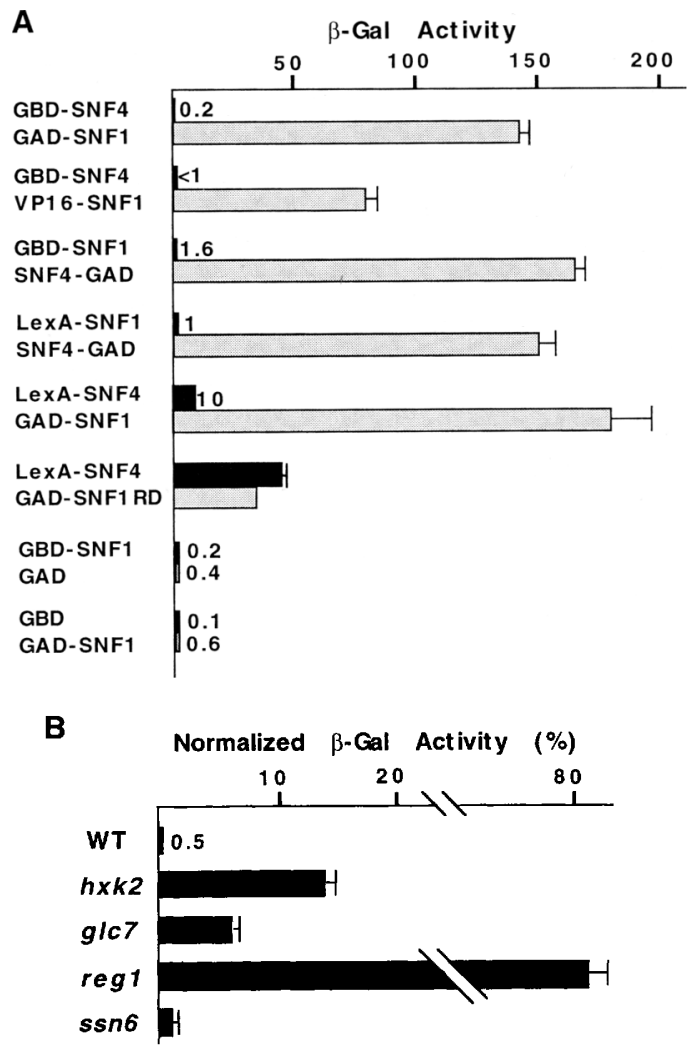

Figure 1. Glucose-regulated interaction of SNF1 and SNF4 in the two-hybrid system. (A) Pairs of DNA-binding and activation domain fusion proteins were expressed from pEE5 and pNI12 (Fields and Song 1989), pSG1, pSD4, pRJ55, pRJ58, pRJ79, pRJ92, pMA424, pLexA(1-202) + PL, pVP16, and pGAD2F (see Table 2). Strains were GGY1::171 and CTY10.5d. Transformants were grown in glucose (solid bar) or Gal/Gly/ETOH plus $0.05 \%$ glucose (shaded bars). Values for $\beta$-galactosidase activity are averages for three to five transformants. Additional control pairs included GBD/SNF4-GAD, LexA-SNF4/GAD, GBD-SNF4/ GAD, GBD/VP16-SNF1, GBD-SNF4/VP16, and LexA-SNF1/ $\mathrm{GAD}$, each yielding negligible activity. Proteins were expressed from the $A D H 1$ promoter or for SNF4-GAD, the SNF4 promoter. Western blots of GAD-SNF1 and LexA $\mathrm{A}_{8}-\mathrm{SNF} 4$ confirmed that expression of the $A D H 1$ promoter is a fewfold higher in glucose (Denis et al. 1983). (B) LexA-SNF1 and SNF4GAD were expressed from pRJ55 and pNI12 (Fields and Song 1989|, respectively. Strains were CTY10.5d (WT) carrying hxk2A::URA3, reg1 $1:: U R A 3$, or ssn $6 \Delta 6:: U R A 3$. MCY3000 (gIc7-T152K) was transformed with the lexAop-lacZ reporter pSH18-18, as was a wild-type control strain. Transformants were grown in glucose or Gal/Gly/ETOH. $\beta$-Galactosidase activity obtained in glucose was normalized to that in Gal/Gly/ ETOH (set at $100 \%$ ); values for Gal/Gly/ETOH were within a factor of two for mutants and wild type (WT). Values are averages of assays of two to six transformants. The effects of these $h \times k 2 \Delta$ and reg1 $1 \Delta$ alleles on glucose repression of gene expression have not been directly compared in this genetic background.

mains and GAL4 (GAD) or VP16 transcription activation domains and using different host strains and reporters. In contrast, no glucose regulation of two-hybrid interac- 
tions was observed for various other fusion pairs (Yang et al. 1994; Tu and Carlson 1995; see below). To confirm that glucose limitation induces SNF1-SNF4 interaction, we also shifted cells expressing LexA-SNF1 and SNF4GAD from $2 \%$ glucose to $0.05 \%$ glucose medium for 3 $\mathrm{hr} ; \beta$-galactosidase activity increased from 1 to 250 units, concomitantly with derepression of the SUC2 (invertase/gene. This differential interaction supports biochemical evidence (Woods et al. 1994) that SNF1 kinase function is controlled by the glucose signal.

\section{Mutation of the upstream regulator HXK2 affects SNF1-SNF4 interaction}

To confirm that the regulated two-hybrid interaction reflects a physiologically relevant response, we tested whether mutations in components of the regulatory pathway affect the interaction in a manner consistent with their genetic relationship to SNF1 (Fig. 1B). We first examined the effects of the negative regulatory gene HXK2, encoding hexokinase PII. Mutation of HXK2 relieves glucose repression of many genes, and an early function in the regulatory pathway has been proposed because hexokinase catalyzes the initial step in glucose catabolism. In addition, genetic analysis indicates that hexokinase PII functions upstream of SNFl (for review, see Johnston and Carlson 1992). To assess regulation of the SNF1-SNF4 interaction by hexokinase, we assayed interaction in an $h \times k 2 \Delta$ mutant. In contrast to the wild type, the mutant showed substantial interaction in glucose (Fig. 1B). Thus, mutation of an upstream negative regulator of the SNF1 pathway has the predicted effect on the SNF1-SNF4 interaction.

Mutation of SSN6 (encoding a transcriptional repressor) also relieves glucose repression of many genes, but SSN6 functions downstream of SNF1 (Schultz and Carlson 1987; Keleher et al. 1992). Appropriately, the SNF1SNF4 interaction was still inhibited by glucose in an ssn 6 mutant. These genetic findings confirm that the glucose regulation of this two-hybrid interaction is functionally significant.

\section{Protein phosphatase 1 regulates the SNF1-SNF4 interaction}

We next tested the effects of protein phosphatase 1 (PP1) on SNF1-SNF4 interaction. Genetic studies have implicated PP1, together with the REG1 targeting subunit, in glucose repression. A specific mutant allele of the PP1 structural gene, glc7-T152K, impairs glucose repression (Tu and Carlson 1994), and deletion of REG1 abolishes glucose repression of all genes tested (Johnston and Carlson 1992; Dombek and Young 1993). Genetic and biochemical evidence indicates that REG1 encodes a regulatory subunit that directs the participation of PP1 in the glucose response (Tu and Carlson 1995). Moreover, double mutant analysis showed that the effects of $81 c 7$ $T 152 \mathrm{~K}$ and reg1A on glucose repression require SNF1 (Neigeborn and Carlson 1987; Tu and Carlson 1994).
These data suggest that PP1/REG1 acts upstream of SNF1, although other roles of PP1 in glucose repression are not excluded.

To examine the possibility that PPI/REG1 regulates SNF1 function, we assayed SNF1-SNF4 interaction in glc7-T152K and reg1A mutants. Both mutations relieved the inhibition by glucose (Fig. 1B). The partial effect of glc7-T152K can be attributed to the fact that this mutation does not abolish GLC7 function, which is essential for viability (Ohkura et al. 1989). Significantly, the effects of these mutations on the SNF1-SNF4 two-hybrid interaction parallel their effects on SUC2 expression: reg1A completely relieves glucose repression of $S U C 2$, whereas glc7-T152K only partially relieves repression (Tu and Carlson 1995). These findings establish that PP1/REG1 functions in the SNF1 pathway and regulates SNF1-SNF4 interaction in response to the glucose signal.

SNF4 binds to the carboxy-terminal regulatory domain of SNF1

The SNF1 kinase has two domains: an amino-terminal kinase domain (SNF1KD, residues 1-391) and a carboxy-terminal regulatory domain (SNFIRD, residues 392-633). To define the region responsible for interaction with SNF4, we expressed partial SNF1 sequences as GAD fusions and tested for interaction with LexA-SNF4 (Fig. 2A). SNF4 did not interact with the kinase domain; subsequent experiments showed that GAD-SNF1KD is expressed and functional. However, SNF4 interacted strongly with residues $392-495$ of the SNF1 regulatory domain, a region that is conserved within the SNF1 kinase family (Fig. 2B). Interestingly, LexA-SNF4 interacted with GAD-SNF1RD in both high and low glucose (Fig. 1A), indicating that the full-length SNF1 is required for glucose-regulated SNF1-SNF4 interaction. The lower levels of $\beta$-galactosidase activity produced by this combination, relative to LexA-SNF4 plus GAD-SNF1, may reflect differences in expression of the fusion proteins as LexA-SNF4 plus GAD-SNF1 ${ }_{392-495}$ produced high activity (see legend to Fig. 2A); however, it is also possible that the presence of the SNF1 kinase domain contributes indirectly in some way to interaction between SNF1 and SNF4.

To determine whether SNF4 binds directly to the SNF1 regulatory domain, we tested purified bacterially expressed proteins for binding in vitro. GST-SNF4 was immobilized on glutathione-Sepharose beads and then incubated with TRX-RD, a fusion between thioredoxin (TRX) and the regulatory domain. Bound proteins were analyzed by Western blotting using a polyclonal antibody against TRX. GST-SNF4 and TRX-RD bound to each other but not to the control proteins GST and TRX (Fig. 3). Thus, SNF4 binds to the SNF1 regulatory domain in vitro. Genetic suppression analysis, described below, confirmed that SNF4 contacts the regulatory domain in vivo. 
A

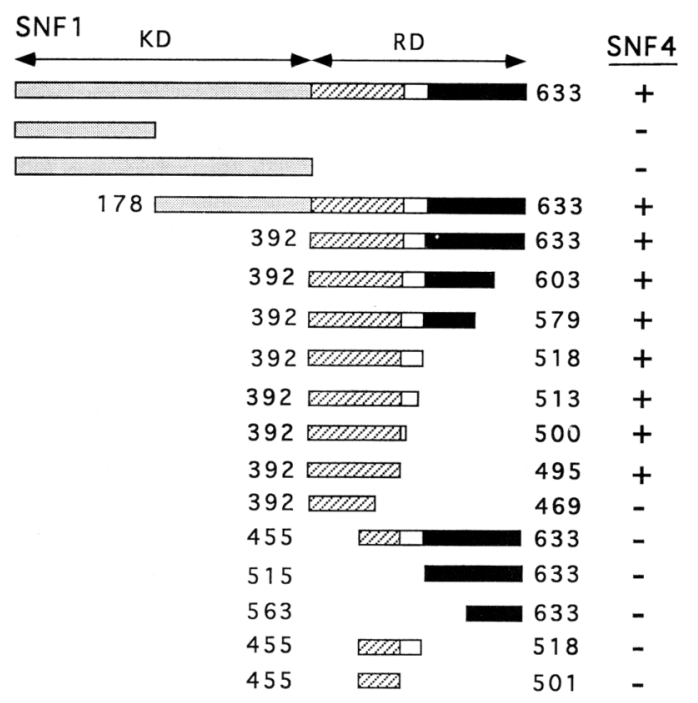

B

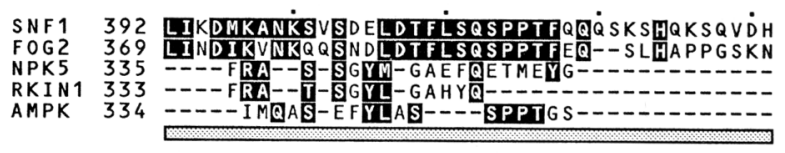

470

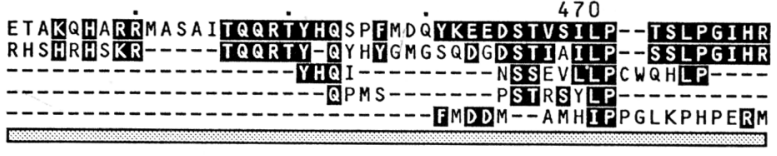

SNF1KD

$+$

ND

ND

$+$

$+$

$+$

$+$

$+$

$+$

-

-

-

$-$

-

-

-

$-$

$-$

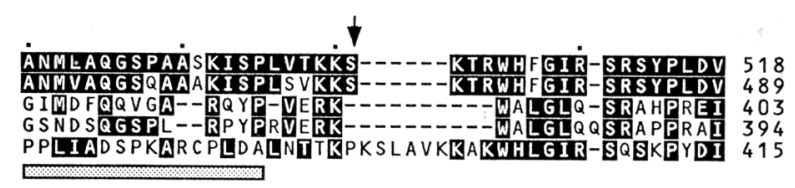

C

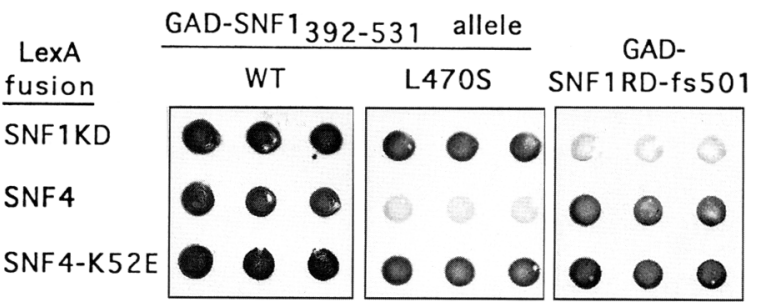

Figure 2. Interaction of SNF1 regulatory domain with SNF4 and the SNF1 kinase domain. $(A)$ SNF1 sequences were expressed as GAD fusions, constructed by cloning restriction fragments and PCR fragments into vectors pACTII (Legrain et al. 1994), pACT (Durfee et al. 1993), and pGADGH (Clontech). GAD-SNF1, GAD-SNF1KD, GAD-SNF1RD, LexA ${ }_{87}-$ SNF4, and LexA $_{87}-$ SNF1KD were expressed from pSG1, pRJ90, pRJ92, pRJ58, and pRJ190, respectively. Transformants $(6-12)$ of strain CTY10.5d were patched onto selective SC-2\% glucose and grown for one day at $30^{\circ} \mathrm{C}$; during this time cells in the patch become limited for glucose. Two-hybrid filter lift assays were then carried out and developed overnight. Because $\beta$-galactosidase is stable, two-hybrid interactions that occur in either high or low glucose are detected. Several combinations were also assayed quantitatively for $\beta$-galactosidase activity. Data for

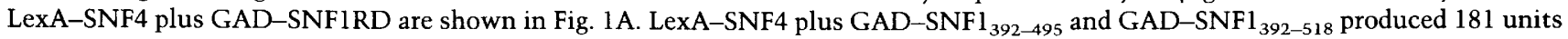
and 41 units, respectively, in high glucose and 464 units and 35 units, respectively, in Gal/Gly/ETOH /values are averages for three to five transformants). (Shaded bar) Kinase domain (KD); (open bar)KD-interacting region; (hatched bar) SNF4-interacting region; (solid bar) carboxy-terminal part of the regulatory domain (RD). (ND) not determined. $(B)$ Conservation of regulatory domain sequences in the SNF1 kinase family. SNFl sequence that interacts with the kinase domain is shown. Bar indicates sequence that interacts with SNF4. Residue L470 is marked, and the position of the fs501 mutation is indicated with an arrow. Kluyveromyces lactis FOG2 (Goffrini et al. 1996), tobacco NPK5 (Muranaka et al. 1994), and rye RKIN1 (Alderson et al. 1991) are functional homologs of SNF1, and FOG2 is required for expression of glucose-repressed genes in K. lactis. AMPK is rat AMP-activated protein kinase /Carling et al. 1994). Identities with SNFl and conserved substitutions are shown in reverse contrast. Numbers indicate amino acids. Dashes indicate gaps. $(C)$ Mutations affecting interaction of the SNFl regulatory domain with SNF4 and the kinase domain are described in the text. Strain CTY10.5d was transformed with plasmids expressing the indicated fusions. Filter lift assays for $\beta$-galactosidase expression were developed for $4 \mathrm{hr}$.

Kinase and regulatory domains of SNF1 interact in high glucose

Evidence that the interaction of LexA-SNF4 and GADSNF1RD is not regulated by glucose (Fig. 1A) raised the possibility that the kinase and regulatory domains of SNF1 interact with one another. We reasoned that if the absence of the kinase domain allows constitutive interaction between the regulatory domain and SNF4, then in high glucose the kinase domain functions to prevent the regulatory domain from binding to SNF4. We therefore examined the interaction of the isolated SNF1 kinase domain with the regulatory domain. LexA-SNF1KD interacted strongly with GAD-SNF1RD (Fig. 2A,C). This interaction is not mediated by SNF4 because it occurs in a snf4 4 mutant and GAD-SNF1KD does not interact with LexA-SNF4 (Fig. 2A).

To test for glucose regulation, the interaction of LexASNF1KD and GAD-SNF1RD was monitored in strain MCY2649 carrying a lexAop-lacZ reporter on pSH18-18. $\beta$-Galactosidase activity was 10 -fold higher in glucose than in galactose/glycerol/ethanol (323 units vs 33 units; values are averages for three transformants). This glucose induction is the opposite regulatory response 
A

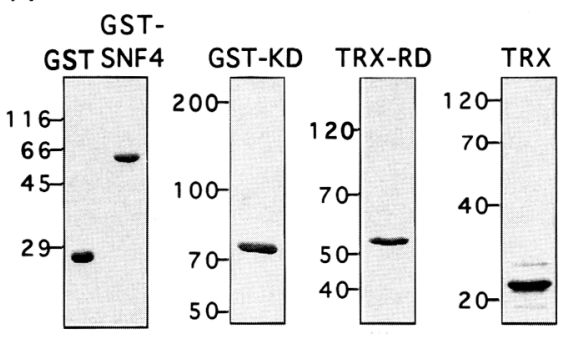

B

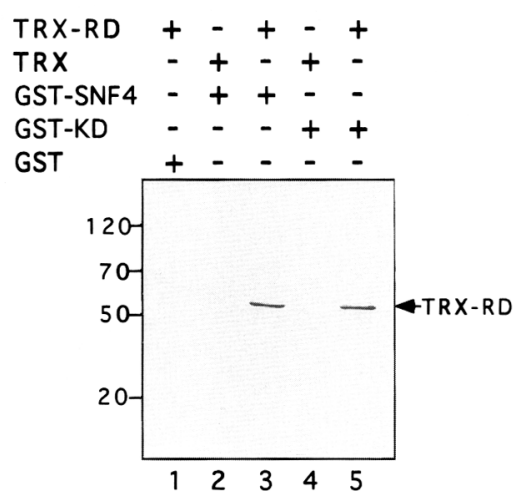

Figure 3. SNF4 and the SNF1 kinase domain bind to the SNF1 regulatory domain in vitro. (A) Purified, bacterially expressed proteins used in the binding assay were separated by electrophoresis on $10 \%$ or $12 \%$ SDS-polyacrylamide gels. Proteins were stained with Coomassie blue. (B) Purified GST-SNF4 1250 $\mathrm{ng})$, GST-KD (250 ng), and GST $(2.5 \mu \mathrm{g})$ were immobilized on glutathione-Sepharose $4 \mathrm{~B}$ beads. Purified TRX-RD (250 ng) and TRX $(2.5 \mu \mathrm{g})$ proteins were allowed to bind to the beads. After extensive washing, bound proteins were electrophoresed on a $10 \%$ SDS-polyacrylamide gel and detected by Western blotting using a polyclonal antibody against TRX. Size standards are indicated in kilodaltons.

from that observed for SNF4 and SNF1, where glucose inhibits interaction.

To obtain biochemical evidence for direct interaction between the SNF1 kinase and regulatory domains, we assayed for binding of purified bacterially expressed proteins in vitro. TRX-RD protein was incubated with GST-KD immobilized on beads, and bound proteins were analyzed by Western blotting using antibody against TRX. TRX-RD bound specifically to GST-KD; TRX and GST proteins served as controls (Fig. 3).

Together, the genetic and biochemical results suggest that the SNF1 kinase domain binds directly to the regulatory domain in high glucose and that binding is diminished when SNF1 function is required for the response to glucose starvation.

SNF4 and the SNF1 kinase domain bind to overlapping sites in the SNF1 regulatory domain

The opposite responses of the SNF1KD-SNF1RD and SNF4-SNF1 interactions to the glucose signal suggest that the kinase domain and SNF4 bind alternatively to the regulatory domain in a glucose-regulated manner. To assess this model, we mapped the sequence in the regulatory domain that is required for binding the kinase domain. The site localizes to residues $392-518$ (Fig. 2A,B), a region that encompasses the SNF4-binding site (residues 392-495).

We then screened for mutations that distinguish between the SNF4- and kinase domain-binding sites. The SNF1 sequence that binds SNF4 was mutagenized by PCR and used to replace the corresponding sequence in GAD-SNF1 $1_{392-531}$, which binds both LexA-SNF1KD and GBD-SNF4. To facilitate identification of the desired mutations, we developed a double two-hybrid strategy. The GAD-SNF1 ${ }_{392-531}$ mutant pool and a plasmid expressing both LexA-SNF1KD and GBD-SNF4 were cotransformed into a strain bearing two reporters, lexAop-lacZ and GAL1-HIS3. Transformants were tested for both blue color and His phenotype, thereby resolving all four possible combinations of interaction in a single screen. We isolated a TTG to TCG mutation, altering Leu470 to Ser, that abolished interaction with SNF4 but maintained interaction with the SNF1 kinase domain (Fig. 2B,C). Thus, although SNF4 and the kinase domain recognize overlapping sequences, their binding sites are distinct.

We then took advantage of the L470S allele to obtain genetic evidence that SNF1 and SNF4 interact directly in vivo. We isolated second-site suppressors in SNF4 that restore interaction with the mutant SNF1 sequence. A plasmid expressing LexA-SNF4 was mutagenized by passage through an Escherichia coli mutator strain, and a two-hybrid screen with GAD-(SNF1-L470S) ${ }_{392-531}$ was performed. We recovered a plasmid with a TTT to CTT mutation altering Lys52 to Glu (LexA-SNF4K52E; Fig. 2Cl. The sequenced fragment was recloned to confirm that this mutation restores interaction. This evidence verifies that SNF4 directly contacts the SNF1 regulatory domain in vivo.

\section{Genetic evidence that SNF4 antagonizes} autoinhibition of the SNF1 kinase activity by the regulatory domain

To explore the functional significance of the interactions of SNF4 and the SNF1 kinase domain with the SNF1 regulatory domain, we turned to genetic approaches. We first analyzed a mutation in the SNF1 regulatory domain, snf1-fs501, which partially suppresses the requirement for SNF4 (Estruch et al. 1992). This frameshift mutation truncates SNF1 within the kinase domain binding site (Fig. 2B). Using a mutant GAD-SNF1RD-fs501 construct, we found that the mutation abolishes interaction with LexA-SNF1 KD (Fig. 2C). Interaction with LexASNF4 was maintained, consistent with the position of fs501 outside the SNF4 recognition sequence, but this interaction is no longer functionally essential.

Similarly, deletion of the regulatory domain from SNF1 also bypasses the requirement for SNF4 (Celenza and Carlson 1989|. Extending this result, we found that 
overexpression of GAD-SNF1KD restores SUC2 expression to a snf4 mutant and does so much more effectively than full-length GAD-SNF1 (Table 1). We also showed that GAD-SNF1KD was expressed at similar levels as GAD-SNF1 (data not shown) and that suppression is dependent on kinase activity. SUC2 expression was still glucose-regulated, indicating that SNF4 is not required for the glucose response in the absence of the regulatory domain and suggesting that the kinase domain is one of the targets of the glucose signal. It remains possible that in the native complex SNF4 is also a target of glucose signals.

Thus, previous studies showed that SNF4 is required for function of the wild-type SNFl kinase in vivo and in vitro (Schuller and Entian 1988; Celenza and Carlson 1989; Celenza et al. 1989; Woods et al. 1994). In contrast, SNF4 is dispensable when the interaction between the SNFl regulatory domain and the kinase domain is abolished. This genetic evidence suggests that SNF4 counteracts autoinhibition of the kinase activity by the SNF1 regulatory domain.

\section{Regulatory interactions in the SNF1 complex are conserved in plants}

Sequence homologs of SNFl have been identified in many plants and mammals, and plant kinases can provide SNF1 function in yeast (Alderson et al. 1991; Muranaka et al. 1994). To assess the conservation of regulatory features of the kinase complex, we examined the tobacco NPK5 kinase, which has $55 \%$ identity to SNF1 overall (Muranaka et al. 1994). First, we constructed a VP16-NPK5 fusion that restores glucose-regulated SUC2 expression in a yeast snf1 1 mutant (Fig. 4A). VP16-NPK5 interacted with LexA-SNF4 in two-hybrid assays, and interaction was inhibited by glucose (Fig. 4B). Furthermore, this interaction is functionally important

Table 1. Deletion of the SNF1 regulatory domain bypasses SNF4 function in SUC2 expression

\begin{tabular}{|c|c|c|c|}
\hline \multirow{2}{*}{$\begin{array}{l}\text { Relevant } \\
\text { genotype }\end{array}$} & \multirow{2}{*}{$\begin{array}{l}\text { Expressed } \\
\text { protein }\end{array}$} & \multicolumn{2}{|c|}{ Invertase activity } \\
\hline & & $\mathrm{R}$ & $\mathrm{D}$ \\
\hline Wild type & GAD & 1.2 & 130 \\
\hline $\operatorname{snf} 4 \Delta$ & GAD & 0.9 & 1.2 \\
\hline $\operatorname{snf} 4 \Delta$ & GAD-SNFI & 1.2 & 10 \\
\hline $\operatorname{snf} 4 \Delta$ & GAD-SNF1KD & 1.4 & 87 \\
\hline $\operatorname{snf} 4 \Delta$ & GAD-SNF1KD-K84R & 0.6 & 0.7 \\
\hline $\operatorname{snf} 1 \Delta \operatorname{snf} 4 \Delta$ & GAD & 0.7 & 1.2 \\
\hline $\operatorname{snf} 1 \Delta \operatorname{snf} 4 \Delta$ & GAD-SNF1KD & 1.3 & 90 \\
\hline
\end{tabular}

Strains were MCY2649, MCY2634, and MCY1793. Expression plasmids were pSG1, pRJ90, and pRJ224. Transformants were grown to mid-log phase in SC-Leu- $2 \%$ glucose (repressed, R) and shifted to SC-Leu- $0.05 \%$ glucose (derepressed, D) for $3 \mathrm{hr}$. Values are averages for two transformants. The control GADSNF1KD-K84R, containing a mutation in the ATP-binding site (Celenza and Carlson 1986), confirmed that kinase activity is required for suppression of $\operatorname{snf} 4$. because VP16-NPK5 did not restore SUC2 expression in a snf4 mutant (Fig. 4A).

We next tested for interaction between the NPK5 kinase and regulatory domains. The fusion proteins LexANPK5KD and GAD-NPK5RD interacted strongly in two-hybrid assays (Fig. 4C). Moreover, cross interaction was readily detected between domains of the yeast and plant proteins. LexA-NPK5KD interacted with GADSNF1RD, and conversely, LexA-SNF1KD interacted with GAD-NPK5RD (Fig. 4C). Thus, both glucose-regulated interaction with SNF4 and specific interactions between the kinase and regulatory domains are conserved from yeast to plants (Fig. 4D).

\section{Discussion}

The SNFl protein kinase is a member of a widely conserved family of kinases that have been implicated in cellular stress responses. In yeast, SNF1 plays a central role in the regulatory response to glucose, and SNF1 kinase activity is required for transcription of glucoserepressed genes in response to glucose deprivation (Johnston and Carlson 1992). Here we have addressed the regulation of SNF1 kinase activity by the glucose signal. We report that the interaction of SNF1 with its activating subunit, SNF4, is strongly regulated by glucose in the two-hybrid system and is appropriately affected by mutations in upstream regulators. These findings, in conjunction with the increase in in vitro activity during glucose starvation (Woods et al. 1994), provide convincing evidence that SNFl activity is controlled by the glucose signal.

Our analysis of this regulated two-hybrid interaction leads us to propose that SNF4 functions in low glucose to counteract autoinhibition of the SNF1 kinase activity by the SNF1 regulatory domain. We show that SNF4 and the SNFl kinase domain bind to overlapping sites in the SNF1 regulatory domain, with opposite regulation by the glucose signal. In vitro binding studies and genetic suppressor analysis confirm that both interactions are direct. Genetic evidence provides insight into the functional significance of these interactions: Deleting SNF4 inactivates the wild-type SNF1 kinase, whereas abolishing interaction of the SNF1 kinase domain with the regulatory domain bypasses the requirement for SNF4 (Celenza and Carlson 1989; this work). We therefore propose that the SNF1 regulatory domain autoinhibits the kinase activity in high glucose, and that SNF4 antagonizes this autoinhibition in low glucose (Fig. 5).

We note an apparent discrepancy between the glucoseregulated interaction of hybrid proteins and previous coimmunoprecipitation and copurification data that the native SNF1 and SNF4 proteins are associated in glucose-grown cells /Celenza et al. 1989; Estruch et al. 1992; Mitchelhill et al. 1994; Stapleton et al. 1994; Yang et al. 1994). From previous biochemical data, we cannot formally exclude that a fraction of the native SNF1 and SNF4 protein in the cell is not present in complexes, and perhaps the two-hybrid system detects interactions representing only this fraction. However, recently we have 
A

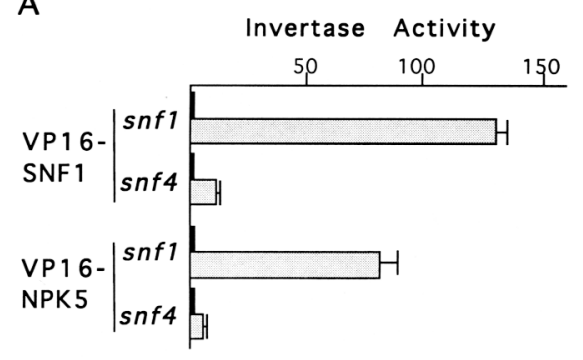

B

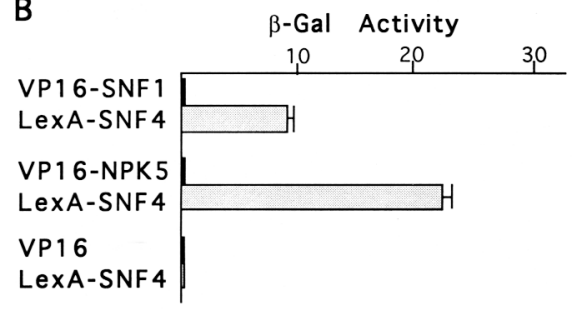

C

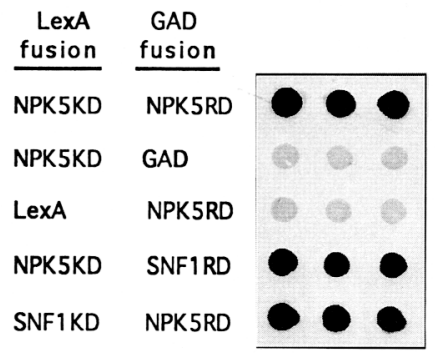

D

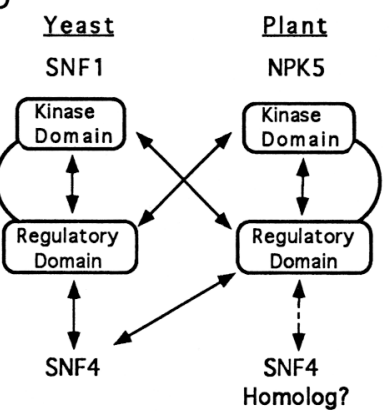

Figure 4. Conserved function and interactions for a plant SNF1 homolog, NPK5. (A) Invertase activity was assayed in strains MCY2916 (snf1A) and MCY2634 (snf4A) expressing the indicated VP16 fusion proteins from $\mathrm{pRJ} 83$ and pRJ79. Transformants were grown in SC-2\% glucose (solid bars) and shifted to $0.05 \%$ glucose (shaded bars) for $3 \mathrm{hr}$. Values are averages for three transformants. VP16 vector controls showed no activity. $(B)$ Two-hybrid interactions between VP16 fusions and LexA-SNF4 (pRJ57) were tested in strain CTY10.5d. $\beta$-Galactosidase activity was assayed after growth in $2 \%$ glucose (solid bars) or Gal/Gly/ETOH (shaded bars). Values are averages for three transformants except for the VP16 controls. LexA controls showed no activity in filter lift assays. In additional control experiments, a fusion to the SSN3/SRB10 cyclin-dependent kinase (Kuchin et al. 1995) did not complement snf1 or interact with SNF4. (C) Two-hybrid interactions of the tobacco NPK5 kinase and regulatory domains were tested in strain CTY10.5d by filter lift assay with color development for $4 \mathrm{hr}$. $(D)$ Conservation of regulatory interactions in the SNFl kinase complex and its homologs. Demonstrated interactions are denoted by solid arrows and predicted interaction by a dashed arrow. shown that SNF4 is quantitatively recovered from extracts of cells grown in either glucose or Gal/Gly/ETOH when SNF1 is affinity-purified on Ni-NTA agarose beads, using its intrinsic histidine tag (R. Jiang and $M$. Carlson, unpubl.). We therefore favor a second explanation. We suggest that another protein(s) interacts with both SNF1 and SNF4, thereby anchoring them into a

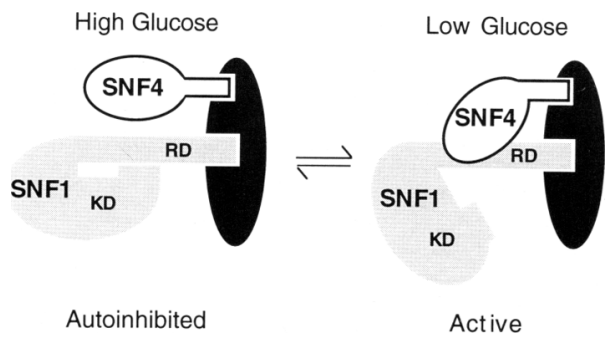

Figure 5. Model for glucose regulation of protein interactions in the SNFl kinase complex. In high glucose, the regulatory domain of SNF1 binds to the SNF1 kinase domain, causing autoinhibition of the kinase activity. When glucose is limiting (low or no glucose), autoinhibition is relieved and SNF4 binds to an overlapping site in the regulatory domain. SNF4 may actively displace the kinase domain and/or stabilize an active conformation. Represented here is a version of the model in which SNF1 and SNF4 are associated in a complex in both high and low glucose with another protein(s) (dark oval) bridging their interaction. complex, within which the interaction of SNF1 and SNF4 varies in response to glucose. The other known components of SNF1 kinase complexes, SIP1, SIP2, and GAL83 (Yang et al. 1992, 1994), are candidates for such bridging proteins. The two-hybrid system may detect only direct interaction of the SNF1 and SNF4 fusion proteins, which occurs in glucose-starved cells, and not indirect interaction bridged by other proteins. We propose that the regulated two-hybrid interactions between SNF1 and SNF4 and between the kinase and regulatory domains reflect conformational changes that occur in the native SNF1 kinase complex in response to the glucose signal (Fig. 5).

Changes in protein-protein interactions within the SNF1 kinase complex could, in principle, be controlled by multiple regulatory inputs to multiple components of the complex. The SNF1 kinase domain is most likely one target of regulatory signals, because expression of the kinase domain in a snf1 snf4 double mutant is sufficient to confer glucose-regulated SUC2 expression (Table 1). One possible mechanism is that the affinity of the kinase domain for the regulatory domain is modulated in response to the glucose signal. When this affinity is reduced, SNF4 may effectively compete for binding, thereby stabilizing the kinase in an active configuration. If there are multiple input signals to the complex, it is also possible that SNF4 is a direct target of regulatory signals and actively displaces the kinase domain.

Our results implicate PP1 and the associated REG1 
targeting subunit in the glucose signaling mechanism. Previous studies showed that PP1/REGl is required for glucose repression of gene expression ( $\mathrm{Tu}$ and Carlson 1994, 1995), and here we identify a mechanism by which PP1/REG1 contributes to repression. Mutations in the cognate structural genes (gIc7-T152K and reg1A) allow SNFl-SNF4 interaction in the presence of glucose, indicating that PP1/REGl acts upstream of the kinase to regulate SNF1-SNF4 interaction. These findings also implicate phosphorylation in regulation of SNFl kinase activity. The conserved Thr210 near subdomain VIII (T loop) is a candidate for phosphorylation, as has been found for other kinases (Hanks and Hunter 1995), because mutation of Thr210 abolishes SNF1 activity (Estruch et al. 1992) and also prevents two-hybrid interaction with SNF4 (R. Jiang and M. Carlson, unpubl.). At present, no upstream kinase has been identified in yeast, and it remains possible that autophosphorylation by SNF1 is important; however, a partially purified mammalian kinase activates AMPK and can reverse inactivation of SNF1 by mammalian protein phosphatase $2 \mathrm{~A}$ (Woods et al. 1994).

An interesting analogy exists between the SNF1 kinase and the related family of calcium/calmodulin (CaM)-dependent kinases (Hanks and Hunter 1995). Calmodulin, in its calcium-bound state, activates CaM kinases by binding to a site overlapping an autoinhibitory sequence in the carboxy-terminal regulatory domain (Kemp and Pearson 1991; Schulman 1993). The crystal structure of CaM kinase I indicates that substantial conformational changes in the kinase are coupled to calmodulin binding (Goldberg et al. 1996). Similarly, activation of the twitchin kinase involves the binding of the calcium effector $\mathrm{S} 100 \mathrm{Al}_{2}$ to part of an autoinhibitory sequence positioned in the active site (Heierhorst et al. 1996). Thus, the regulated interactions that we detect in the SNF1 complex, and the proposed resulting conformational change, appear to represent a variation of a general regulatory mechanism that has been adapted for use in diverse signaling systems.

In the last few years the cloning of SNF1 homologs from diverse organisms has revealed that SNFl is a member of a large family of kinases. Moreover, the regulatory interactions that we have detected within the yeast SNF1 kinase complex appear to represent conserved features of complexes in the SNF1 family (Fig. $4 \mathrm{D}$ |. We demonstrate glucose-regulated interaction between the plant homolog NPK5 and the yeast SNF4 protein and also cross-species interactions between the NPK5 and SNF1 kinase and regulatory domains. This conservation most likely also extends to mammals, as mammalian homologs of SNF4 and SIP2 are associated with AMPK, a SNF1 homolog (Stapleton et al. 1994; Woods et al. 1996).

Do SNF1 kinase homologs serve similar regulatory functions in all eukaryotes? This seems likely for the plant homologs, as they provide SNF1 function in yeast; moreover, glucose and other sugars are known to repress transcription of photosynthetic genes in plants (Jang and Sheen 1994). The mammalian homolog AMPK plays a central role in regulating lipid metabolism and in the cellular stress response (Corton et al. 1994; Hardie et al. 1994). A role for AMPK in regulating gene expression has not yet been explored. The yeast SNF1 kinase does not appear to be activated by AMP, but like AMPK, SNF1 phosphorylates and inhibits activity of acetyl-CoA carboxylase (Mitchelhill et al. 1994; Woods et al. 1994), suggesting a similar role in regulating fatty acid biosynthesis. Moreover, SNF1 is involved in responses to other stresses besides glucose starvation and is known to affect thermotolerance, carbohydrate storage, sporulation, and peroxisomal assembly (Thompson-Jaeger et al. 1991; Simon et al. 1992). Further characterization of these functions of SNF1 will likely extend the parallels with AMPK.

Thus, both the components of the SNF1 kinase complex, including SNF4 and SIP2, and many of its regulatory functions have been conserved in evolution. The signaling inputs into this pathway, however, appear to vary in different organisms. We suggest that SNF1 represents a kinase pathway that has been adapted for a broad range of responses to environmental and nutritional stresses.

\section{Materials and methods}

Plasmids, strains, and genetic methods

Plasmids were constructed using standard methods (Sambrook et al. 1989) and are listed in Table 2 or described in the text. pSH18-18 was a gift of R. Brent (Massachusetts General Hospital, Boston). S. cerevisiae strains were CTY10.5d (MATa ade2 his3 leu2 trp1 gal80 gal4 lexAop-lacZ) (gift of R. Sternglanz,

Table 2. Plasmids used in this study

\begin{tabular}{|c|c|}
\hline Name & Description \\
\hline pRJ55 & LexA-SNF1 in vector $\mathrm{pLexA}(1-202)+\mathrm{PL}$ \\
\hline pRJ57 & LexA-SNF4 in vector $\mathrm{pLexA}(1-202)+\mathrm{PL}$ \\
\hline pRJ58 & LexA $_{87}$-SNF4 in vector $\mathrm{pSH} 2-1$ \\
\hline pRI79 & VP16-SNF1 in vector pVP16 \\
\hline pRJ83 & VP16-NPK5 in vector pVP16 \\
\hline pRJ90 & GAD-SNFIKD(BamHI-BcII fragment) in pACTII \\
\hline pRJ92 & GAD-SNFIRD (BclI-BamHI fragment $)$ in pACTII \\
\hline pRJ98 & GAD-SNF1RD-fs501 in vector pACTII \\
\hline pRJ113 & GAD-SNFl ${ }_{392-531}$ in vector $\mathrm{pACTII}$ \\
\hline pRJ190 & LexA $_{87}$-SNF1KD (BamHI-Bcll fragment) in pSH2-1 \\
\hline pRJ224 & GAD-SNF1KD-K84R in vector pACTII \\
\hline pRJ273 & LexA $_{87}-\mathrm{NPK} 5 \mathrm{KD}$ (residues $1-334$ ) in vector $\mathrm{pSH} 2-1$ \\
\hline pRJ277 & GAD-NPK5RD (residues 335-511) in vector pACTII \\
\hline pRJ287 & GAD-(SNF1-L47OS $)_{392-531}$ in vector pACTII \\
\hline pRJ292 & $\mathrm{LexA}_{87}$-SNF4-K52E in vector $\mathrm{pSH} 2-1$ \\
\hline pSG1 & GAD-SNF1 in vector pGAD2F \\
\hline pSD4 & GBD-SNF4 in vector pMA424 \\
\hline
\end{tabular}

pSH1 and pSD4 were gifts of $\mathrm{Z}$. Xue and T. Melese /Columbia University, New York, NY). pRJ83, 273, and 277 were constructed using PCR with an NPK5 clone (Muranaka et al. 1994) as template. pRJ98 contains codons 392-633 on a BamHI-EcoRI PCR fragment. Vectors: pVP16 (Vojtek et al. 1993), pACTII (gift of S. Elledge) (Legrain et al. 1994), pGAD2F (Chien et al. 1991), pMA424 (Ma and Ptashne 1987), pSH2-1 (Hanes and Brent 1989), and pLexA(1-202) + PL (Ruden et al. 1991). 
State University of New York, Stony Brook), GGY1::151 (his3 leu2 gal80 gal4 GAL1-lacZ) (Gill and Ptashne 1987), MCY1793

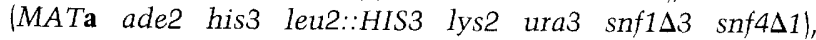

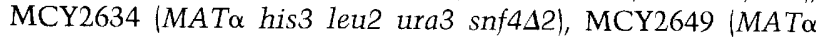
his3 leu2 ura3), MCY2916 (MATo his3 leu2 ura3 snf1D10), and MCY3000 (MATa his3 leu2 lys2 trp1 ura3 glc7-T152K). Alleles are his3-4200, lys2-801, ura3-52, and leu2-3,112 except where noted. Standard genetic methods were followed, and yeast cultures were grown in synthetic complete $(\mathrm{SC})$ medium lacking appropriate amino acids to maintain selection for plasmids (Rose et al. 1990).

\section{$\beta$-Galactosidase and invertase assays}

To test $\beta$-galactosidase expression, transformants were patched onto SC-His-Leu- $2 \%$ glucose plates and incubated one day at $30^{\circ} \mathrm{C}$, and then filter lift assays were performed (Yang et al. 1992). For quantitation, transformants were grown to mid-log phase in selective SC- $2 \%$ glucose (Glu) or SC containing $2 \%$ galactose, glycerol, ethanol and $0.05 \%$ glucose /Gal/Gly/ $\mathrm{ETOH}$ ), unless otherwise indicated. $\beta$-Galactosidase activity was assayed in permeabilized cells and expressed in Miller units. Secreted invertase activity was assayed in whole cells (Yang et al. 1994) and is expressed as micromole of glucose released/min per $100 \mathrm{mg}$ cells (dry weight).

\section{Double two-hybrid screen}

SNFl codons 392-495 were PCR-mutagenized (Leung et al. 1989; Zhou et al. 1991), digested with BclI plus PvuII (sites at codons 392 and 489, respectively), and used to replace the corresponding sequence between the BamHI and PvuII sites in GAD-SNF $1_{392-531}$ (pRJ113). The resulting pool of mutants was cotransformed with a plasmid expressing both LexA-SNF1KD

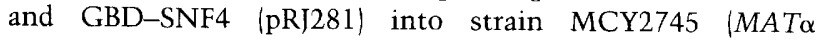
LYS2::GAL1-HIS3 URA3::lexAop-lacZ ade2 his3 leu2 met trp1 gal4 gal80). MCY2745 carries an integrative version of $\mathrm{pSH} 18$ 18 (pRJ149). Transformants were tested for blue color and His phenotype (by replica-plating onto SC-Trp-Leu-His plates containing $5 \mathrm{~mm}$ 3-amino-1,2,4 triazole), thereby assessing both interactions. About $10^{5}$ transformants were screened. Mutations were identified by DNA sequencing (codons 392-531). The mutation L470S was recovered once alone and six times in combination with other mutations.

\section{Isolation of SNF4-K52E suppressor}

A mutant library was constructed by passage of a plasmid expressing LexA-SNF4 (pRJ58) through an E. coli mutator strain (XL-1 Red, Stratagene). To identify mutations restoring twohybrid interaction with GAD-(SNF1-L470S $)_{392-531}, 2 \times 10^{6}$ transformants of strain CTY10.5d were screened for $\beta$-galactosidase expression by filter lift assay. The SNF4-K52E mutation was identified by DNA sequencing of both strands. Sequenced DNA was recloned into vector pSH2-1 (Hanes and Brent 1989) and the resulting LexA-SNF4-K52E fusion was shown to interact with GAD-(SNF1-L470S 392-531 but not with $\mathrm{GAD}$ alone, by filter lift assays.

\section{Protein expression and in vitro binding assays}

SNF4 and the SNF1 kinase domain were cloned into pGEX-3X (Pharmacia) as glutathione $S$-transferase (GST) fusions (pRJ31 and pRJ205, respectively). The regulatory domain was cloned into pET-32a $(+)$ (Novagen) to express a thioredoxin (TRX) fusion with an amino-terminal $6 \mathrm{xHis}$ tag (pRJ255). E. coli strains XL-1 blue, GM48 (Stratagene), and BL21 (DE3) (Novagen) were used for expression, which was induced by addition of IPTG (1 $\mathrm{mm}$ final) to the culture. Bacterial pellets were resuspended in buffer ST (10 mM Tris- $\mathrm{HCl}$ at $\mathrm{pH} 8.0,150 \mathrm{~mm} \mathrm{NaCl})$ with 100 $\mu \mathrm{g} / \mathrm{ml}$ lysozyme and sonicated on ice (Frangioni and Neel 1993). After spinning at $12,000 \mathrm{rpm}$ for $15 \mathrm{~min}$ in a Sorvall SS-34 rotor, the supernatants were collected and incubated with glutathione-Sepharose 4B beads (Pharmacia) (GST fusions) or with $\mathrm{Ni}^{2+}$-NTA-agarose beads (Qiagen) (TRX fusions) at $4^{\circ} \mathrm{C}$ for $1 \mathrm{hr}$. Beads were poured into a column and washed sequentially with buffers ST and ST plus $0.5 \mathrm{~m} \mathrm{NaCl}$. GST fusions were eluted with buffer containing $50 \mathrm{~mm}$ Tris- $\mathrm{HCl}$ at $\mathrm{pH} 7.5,20 \mathrm{~mm}$ glutathione (reduced), and TRX fusions were eluted with ST containing 5, 20, 50, 200, and $500 \mathrm{~mm}$ imidazole. Fractions (1 ml) were collected and analyzed by $10 \%$ SDS-PAGE. Peak fractions for each protein were pooled and dialyzed against ST buffer, concentrated using centricon (Amicon) and stored as aliquots in ST plus $10 \%$ glycerol at $-80^{\circ} \mathrm{C}$.

For in vitro binding, purified GST-SNF4, GST-KD, and GST proteins were immobilized on glutathione-Sepharose 4B beads $(50 \mu 1)$. Purified TRX-RD and TRX proteins were added and allowed to bind at $4^{\circ} \mathrm{C}$ for $2 \mathrm{hr}$ in buffer ST $(500 \mu \mathrm{l})$. After extensive washing with buffer ST ( $10 \mathrm{ml})$, the beads were boiled in SDS sample buffer and loaded onto a 10\% SDS-polyacrylamide gel. Bound proteins were detected by Western analysis using a polyclonal antibody against TRX (Santa Cruz Biotech) and enhanced chemiluminescence with ECL reagents (Amersham).

\section{Acknowledgments}

We thank Pascale Lesage and E. Jane Hubbard for the initial observations on glucose regulation of the two-hybrid interaction of SNF1 and SNF4. We thank R. Brent, Z. Xue, T. Melese, and $\mathrm{Y}$. Machida for providing reagents and sharing information prior to publication. This work was supported by grant GM34095 from the National Institutes of Health to M.C.

The publication costs of this article were defrayed in part by payment of page charges. This article must therefore be hereby marked "advertisement" in accordance with 18 USC section 1734 solely to indicate this fact.

\section{References}

Aguan, K., J. Scott, C.G. See, and N. Sarkar. 1994. Characterization and chromosomal localization of the human homologue of a rat AMP-activated protein kinase-encoding gene: A major regulator of lipid metabolism in mammals. Gene 149: $345-350$.

Alderson, A., P.A. Sabelli, J.R. Dickinson, D. Cole, M. Richardson, M. Kreis, P.R. Shewry, and N.G. Halford. 1991. Complementation of snf1, a mutation affecting global regulation of carbon metabolism in yeast, by a plant protein kinase cDNA. Proc. Natl. Acad. Sci. 88: 8602-8605.

Carling, D., K. Aguan, A. Woods, A.J.M. Verhoeven, R.K. Beri, C.H. Brennan, C. Sidebottom, M.D. Davison, and J. Scott 1994. Mammalian AMP-activated protein kinase is homologous to yeast and plant protein kinases involved in the regulation of carbon metabolism. J. Biol. Chem. 289: 1144211448.

Celenza, J.L. and M. Carlson. 1986. A yeast gene that is essential for release from glucose repression encodes a protein kinase. Science 233: 1175-1180.

. 1989. Mutational analysis of the Saccharomyces cerevisiae SNF1 protein kinase and evidence for functional interaction with the SNF4 protein. Mol. Cell. Biol. 9: 5034 
5044.

Celenza, J.L., F.J. Eng, and M. Carlson. 1989. Molecular analysis of the SNF4 gene of Saccharomyces cerevisiae: Evidence for physical association of the SNF4 protein with the SNF1 protein kinase. Mol. Cell. Biol 9: 5045-5054.

Chien, C., P.L. Bartel, R. Sternglanz, and S. Fields. 1991. The two-hybrid system: A method to identify and clone genes for proteins that interact with a protein of interest. Proc. Natl. Acad. Sci. 88: 9578-9582.

Corton, J.M., J.G. Gillespie, and D.G. Hardie. 1994. Role of the AMP-activated protein kinase in the cellular stress response. Curr. Biol. 4: 315-324.

Dale, S., W. Wilson, A. Edelman, and D.G. Hardie. 1995. Similar substrate recognition motifs for mammalian AMP-activated protien kinase, higher plant HMG-CoA reductase kinase-A, yeast SNF1, and mammalian calmodulin-dependent protein kinase I. FEBS Lett. 361: 191-195.

Denis, C.L., J. Ferguson, and E.T. Young. 1983. mRNA levels for the fermentative alcohol dehydrogenase of Saccharomyces cerevisiae decrease upon growth on a nonfermentable carbon source. I. Biol. Chem. 258: 1165-1171.

Dombek, K.M. and E.T. Young. 1993. ADH2 expression is repressed by REGl independently of mutations that alter the phosphorylation of the yeast transcription factor ADR1. Mol. Cell. Biol. 13: 4391-4399.

Durfee, T., K. Becherer, P.-L. Chen, S.-H. Yeh, Y. Yang, A. Kilburn, W.-H. Lee, and S. Elledge. 1993. The retinoblastoma protein associates with the protein phosphatase type 1 catalytic subunit. Genes \& Dev. 7: 555-569.

Erickson, J.R. and M. Johnston. 1993. Genetic and molecular characterization of GAL83: Its interaction and similarities with other genes involved in glucose repression in Saccharomyces cerevisiae. Genetics 135: 655-664.

Estruch, F., M.A. Treitel, X. Yang, and M. Carlson. 1992. N-terminal mutations modulate yeast SNF1 protein kinase function. Genetics 132: 639-650.

Fields, S. and O. Song. 1989. A novel genetic system to detect protein-protein interactions. Nature 340: 245-246.

Frangioni, J.V. and B.G. Neel. 1993. Solubilization and purification of enzymatically active glutathione S-tranferase (pGEX) fusion proteins. Anal. Biochem. 210: 179-187.

Gao, G., J. Widmer, D. Stapleton, T. Teh, T. Cox, B. Kemp, and L. Witters. 1995. Catalytic subunits of the porcine and rat 5 -AMP-activated protein kinase are members of the SNFl protein kinase family. Biochim. Biophys. Acta 1266: 73-82.

Gill, G. and M. Ptashne. 1987. Mutants of GAL4 protein altered in an activation function. Cell 51: 121-126.

Goffrini, P., A. Ficarelli, C. Donnini, T. Lodi, P.P. Puglisi, and I. Ferrero. 1996. FOG1 and FOG2 genes, required for the transcriptional activation of glucose-repressible genes of Kluyveromyces lactis, are homologous to GAL83 and SNF1 of Saccharomyces cerevisiae. Curr. Genet. 29: 316-326.

Goldberg, J., A.C. Nairn, and J. Kuriyan. 1996. Structural basis for the autoinhibition of calcium/calmodulin-dependent protein kinase I. Cell 84: 875-887.

Guo, S. and K.J. Kemphues. 1995. par-1, a gene required for establishing polarity in C. elegans embryos, encodes a putative ser/thr kinase that is asymmetrically distributed. Cell 81: 611-620.

Halford, N.G., J. Vicente-Carbajosa, P.A. Sabelli, P.R. Shewry, U. Hannappel, and M. Kreis. 1992. Molecular analyses of a barley multigene family homologous to the yeast protein kinase gene SNF1. Plant I. 2: 791-797.

Hanes, S.D. and R. Brent. 1989. DNA specificity of the bicoid activator protein is determined by homeodomain recognition helix residue 9. Cell 57: 1275-1283.
Hanks, S.K. and T. Hunter. 1995. The eucaryotic protein kinase superfamily. In The protein kinase factsbook: Protein-serine kinases (ed. G. Hardie and S. Hanks), pp. 7-47. Academic Press, San Diego, CA.

Hardie, D.G. 1992. Regulation of fatty acid and cholesterol metabolism by the AMP-activated protein kinase. Biochim. Biophys. Acta. 1123: 231-238.

Hardie, D.G. 1994. Ways of coping with stress. Nature 370: $599-600$.

Hardie, D.G., D. Carling, and N. Halford. 1994. Role of the Snfl/Rkin1/AMP-activated protein kinase family in the response to environment and nutritional stress. Semin. Cell Biol. 5: 409-4l6.

Heierhorst, J., B. Kobe, S.C. Feil, M.W. Parker, G.M. Benian, K.R. Weiss, and B. Kemp. 1996. $\mathrm{Ca}^{2+} / \mathrm{S} 100$ regulation of giant protein kinases. Nature 380: 636-639.

Jang, J.-C. and J. Sheen. 1994. Sugar sensing in higher plants. Plant Cell 6: 1665-1679.

Johnston, M. and M. Carlson. 1992. Regulation of carbon and phosphate utilization. In The molecular and cellular biology of the yeast Saccharomyces: Gene expression led. E.W. Jones, J.R. Pringle and J.R. Broach), Vol. 2, pp. 193-281. Cold Spring Harbor Laboratory Press, Cold Spring Harbor, NY.

Keleher, C.A., M.J. Redd, J. Schultz, M. Carlson, and A.D. Johnson. 1992. Ssn6-Tupl is a general repressor of transcription in yeast. Cell 68: 708-719.

Kemp, B.E. and R.B. Pearson. 1991. Intrasteric regulation of protein kinases and phosphatases. Biochim. Biophys. Acta 1094: 67-76.

Kuchin, S., P. Yeghiayan, and M. Carlson. 1995. Cyclin-dependent protein kinase and cyclin homologs SSN3 and SSN8 contribute to transcriptional control in yeast. Proc. Natl. Acad. Sci. 92: 4006-4010.

Legrain, P., M.-C. Dokhelar, and C. Transy. 1994. Detection of protein-protein interactions using different vectors in the two-hybrid system. Nucleic Acids Res. 22: 3241-3242.

Le Guen, L., M. Thomas, M. Bianchi, N.G. Halford, and M. Kreis. 1992. Structure and expression of a gene from Arabidopsis thaliana encoding a protein related to SNF1 protein kinase. Gene 120: 249-254.

Leung, D., E. Chen, and D. Goeddal. 1989. A method for random mutagenesis of a defined DNA segment using a modified polymerase chain reaction. Technique 1: 11-15.

Ma, J. and M. Ptashne. 1987. A new class of yeast transcriptional activators. Cell 51: 113-119.

Mitchelhill, K.I., D. Stapleton, G. Gao, C. House, B. Michell, F. Katsis, L.A. Witters, and B.E. Kemp. 1994. Mammalian AMP-activated protein kinase shares structural and functional homology with the catalytic domain of yeast Snfl protein kinase. J. Biol. Chem. 269: 2361-2364.

Muranaka, T., H. Banno, and Y. Machida. 1994. Characterization of tobacco protein kinase NPK5, a homolog of Saccharomyces cerevisiae SNF1 that constitutively activates expression of the glucose-repressible SUC2 gene for a secreted invertase of S. cerevisiae. Mol. Cell. Biol. 14: 2958-2965.

Neigeborn, L. and M. Carlson. 1987. Mutations causing constitutive invertase synthesis in yeast: Genetic interactions with snf mutations. Genetics 115: 247-253.

Ohkura, H., N. Kinoshita, S. Minatani, S. Toda, and M. Yanagida. 1989. The fission yeast dis $2^{+}$gene required for chromosome disjoining encodes one of two putative type 1 protein phosphatases. Cell 57: 997-1007.

Rose, M.D., F. Winston, and P. Hieter. 1990. Methods in yeast genetics: A laboratory course manual. Cold Spring Harbor Laboratory Press, Cold Spring Harbor, NY.

Ruden, D.M., J. Ma, Y. Li, K. Wood, and M. Ptashne. 1991. 
Generating yeast transcriptional activators containing no yeast protein sequences. Nature 350: 250-252.

Ruiz, J., F. Conlon, and E. Robertson. 1994. Identification of novel protein kinases expressed in the myocardium of the developing mouse heart. Mech. Dev. 48: 153-164.

Sambrook, J., E.F. Fritsch, and T. Maniatis. 1989. Molecular cloning: A laboratory manual. Cold Spring Harbor Laboratory Press, Cold Spring Harbor, NY.

Schuller, H.J. and K.-D. Entian. 1987. Isolation and expression analysis of two yeast regulatory genes involved in the derepression of glucose-repressible enzymes. Mol. Gen. Genet. 209: 366-373.

- 1988. Molecular characterization of yeast regulatory gene $C A T 3$ necessary for glucose derepression and nuclear localization of its product. Gene 67: 247-257.

Schulman, H. 1993. The multifunctional $\mathrm{Ca}^{2+} / \mathrm{calmodulin}-\mathrm{de}-$ pendent protein kinases. Curr. Biol. 5: 247-253.

Schultz, J. and M. Carlson. 1987. Molecular analysis of SSN6, a gene functionally related to the SNF1 protein kinase of Saccharomyces cerevisiae. Mol. Cell. Biol 7: 3637-3645.

Simon, M., M. Binder, G. Adam, A. Hartig, and H. Ruis. 1992. Control of peroxisome proliferation in Saccharomyces cerevisiae by ADR1, SNF1 (CAT1, CCR1) and SNF4 (CAT3). Yeast 8: 303-309.

Stapleton, D., G. Gao, B. Michell, J. Widmer, K. Mitchehill, T. Teh, C. House, L. Witters, and B. Kemp. 1994. Mammalian 5 '-AMP-activated protein kinase non-catalytic subunits are homologs of proteins that interact with yeast Snfl protein kinase. I. Biol. Chem. 269: 29343-29346.

Thompson-Jaeger, S., J. Francois, J.P. Gaughran, and K. Tatchell. 1991. Deletion of $S N F 1$ affects the nutrient response of yeast and resembles mutations which activate the adenylate cyclase pathway. Genetics 129: 697-706.

Tu, J. and M. Carlson. 1994. The GLC7 type 1 protein phosphatase is required for glucose repression in Saccharomyces cerevisiae. Mol. Cell. Biol. 14: 6789-6796.

- 1995. REG1 binds to protein phosphatase type 1 and regulates glucose repression in Saccharomyces cerevisiae. EMBO /. 14: 5939-5946.

Vojtek, A.B., S.M. Hollenberg, and J.A. Cooper. 1993. Mammalian Ras interacts directly with the serine/threonine kinase Raf. Cell 74: 205-214.

Woods, A., M.R. Munday, J. Scott, X. Yang, M. Carlson, and D. Carling. 1994. Yeast SNF1 is functionally related to mammalian AMP-activated protein kinase and regulates acetylCoA carboxylase in vivo. J. Biol. Chem. 269: 19509-19516.

Woods, A., P.C. Cheung, F.C. Smith, M.D. Davison, J. Scott, R.K. Beri, and D. Carling. 1996. Characterization of AMPactivated protein kinase $\beta$ and $\gamma$ subunits. I. Biol. Chem. 271: 10282-10290.

Yang, X., E.J.A. Hubbard, and M. Carlson. 1992. A protein kinase substrate identified by the two-hybrid system. Science 257: 680-682.

Yang, X., R. Jiang, and M. Carlson. 1994. A family of proteins containing a conserved domain that mediates interaction with the yeast SNFl protein kinase complex. EMBO $J$. 13: $5878-5886$.

Zhou, Y., X. Zhang, and R.H. Ebright. 1991. Random mutagenesis of gene-sized DNA molecules by use of PCR with Taq DNA polymerase. Nucleic Acids Res. 19: 6052. 


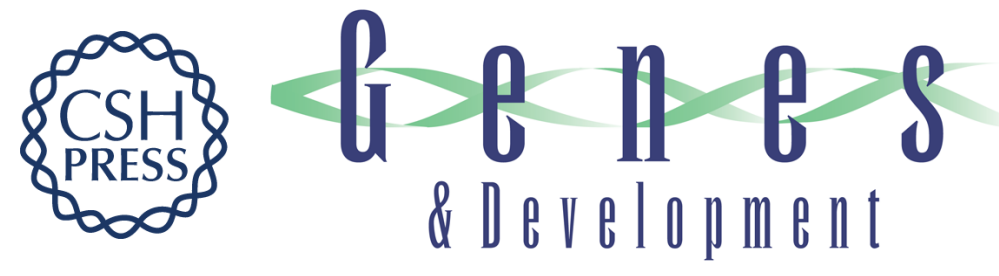

\section{Glucose regulates protein interactions within the yeast SNF1 protein kinase complex.}

$\mathrm{R}$ Jiang and $\mathrm{M}$ Carlson

Genes Dev. 1996, 10:

Access the most recent version at doi:10.1101/gad.10.24.3105

References This article cites 56 articles, 22 of which can be accessed free at:

http://genesdev.cshlp.org/content/10/24/3105.full.html\#ref-list-1

License

Email Alerting

Service

Receive free email alerts when new articles cite this article - sign up in the box at the top right corner of the article or click here.

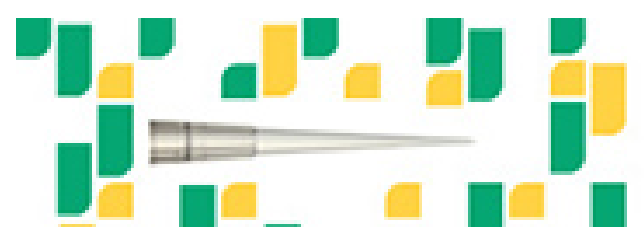

Focused on your science.

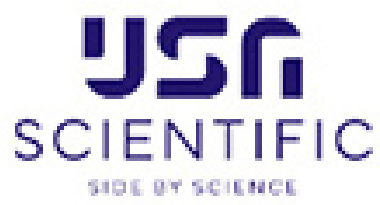

Copyright (c) Cold Spring Harbor Laboratory Press 